\title{
Publisher Correction: Queer geoscientists need more than visibility
}

Robert N. Ulrich (D)

Correction to: Nature Reviews Earth \& Environment (2021) https://doi.org/10.1038/s43017-021-00188-2, published online 14 June 2021.

The original version of this article omitted the image credit line which should have read: "Credit: Image courtesy of Ashley Kruythoff”. This error has been corrected in the HTML and PDF versions of the article.

https://doi.org/10.1038/s43017-021-00193-5 I Published online 18 June 2021

๑) Springer Nature Limited 2021 